\title{
SOINS INFIRMIERS POUR LES PATIENTS PIED DIABÉTIQUE
}

\section{ARTICLE ORIGINAL}

CUNHA, Amanda Priscilla da ${ }^{1}$

CUNHA, Amanda Priscilla da. Soins infirmiers pour les patients pied diabétique. Revista Científica Multidisciplinar Núcleo do Conhecimento. Année. 06, Ed. 12, vol. 12, p. 111 à 126. Décembre 2021. ISSN: 2448-0959, Lien d'accès: https://www.nucleodoconhecimento.com.br/sante/pied-diabetique, $\quad$ DOI: 10.32749/nucleodoconhecimento.com.br/sante/pied-diabetique

\section{RÉSUMÉ}

Introduction: Le pied diabétique également appelé par un état physiopathologique multiforme est caractérisé par des lésions qui proviennent des pieds de la personne atteinte de diabète sans traitement adéquat et soins spécifiques. On estime que l'incidence de l'ulcère du pied diabétique atteint 6,3\% des patients diabétiques et sa prévalence approche les $10 \%$, et les personnes ayant un faible statut socioéconomique sont les plus grandes victimes. Compte tenu de ces preuves, le travail est basé sur la question problématique suivante: Quels soins les infirmières développent-elles chez les patients ayant des pieds diabétiques? Objectif général : Mettre en évidence les soins du pied diabétique liés à la pratique des infirmières. Méthodologie : Une revue intégrative a été menée dans la base de données bibliographiques spécialisées en soins infirmiers (BDENF), la littérature latinoaméricaine et caribéenne en sciences de la santé (LILACS) et la Scientific Electronic Library Online (SCIELO) et a donné lieu à sept articles. Résultats: Les études ont montré comment les soins des infirmières avec l'éducation à la santé du pied diabétique, la formation continue et permanente, les techniques d'évaluation du pied diabétique qui impliquent des examens physiques et les soins personnels des patients atteints de pieds diabétiques. II est renforcé que ces soins devraient être

\footnotetext{
${ }^{1}$ Master en gestion hospitalière et systèmes de santé, infirmière complète en soins infirmiers. ORCID: 0000001-5456-1848.

RC: 105164

Disponible en: https://www.nucleodoconhecimento.com.br/sante/pied-diabetique
} 
effectués en priorité par les infirmières, puisque les infirmières ont des soins spécifiques et qualifiés au patient dans leurs principales attributions. Conclusion : ॥ est donc conclu que l'étude a identifié plusieurs formes de soins dispensés par les infirmières et que toutes ces formes sont importantes pour la continuité du traitement et la prévention des complications, cependant, il appartient à l'infirmière d'être un professionnel qui valorise la qualité de ses soins en fonction de concepts théoriques qui conduisent à une formation fréquente.

Mots-clés: Soins aux patients, Diabète sucré, Pied diabétique.

\section{INTRODUCTION}

Le diabète sucré (DS) est une maladie développée dans le système endocrinien et/ou un trouble métabolique chronique, représentant un grave problème de santé mondial et ayant pour principale caractéristique l'hyperglycémie, atteignant une glycémie supérieure à $126 \mathrm{mg} / \mathrm{dL}$ à jeun ou >200 mg/dL au stade post-prandial, et qui peut avoir été associée à une résistance à l'insuline, et qui peut avoir été associée à une résistance à l'insuline, une sécrétion insuffisante d'insuline ou une sécrétion excessive de glucagon (ASCHNER et al., 2016).

Par conséquent, l'hyperglycémie provoque une incapacité totale ou partielle de production d'insuline, selon la Société brésilienne du diabète (SBD), l'American Diabetes Association (ADA) et l'Organisation mondiale de la santé (OMS) DS est divisé en quatre groupes: diabète sucré de type 1, diabète sucré de type 2, diabète sucré gestationnel et autres types spécifiques de diabète (OMS, 1999; SBD, 2017; ADA 2017).

SBD décrit les facteurs de risque de DS, qui sont cités comme suit: diagnostic de prédiabète - diminution de la tolérance au glucose ou altération du glucose à jeun; hypertension; hypercholestérolémie ou changements dans le taux de triglycérides dans le sang; surpoids et obésité, surtout si la graisse est concentrée autour de la taille; antécédents familiaux; bébé pesant plus de quatre kilos ou diabète gestationnel; syndrome des ovaires polykystiques; diagnostic de certains troubles 
psychiatriques, tels que la schizophrénie, la dépression, le trouble bipolaire et l'utilisation de médicaments psychotropes; d'autres (SBD, 2018).

Comme il s'agit d'une maladie qui touche environ $3 \%$ de la population mondiale et avec une prévalence approximative de $6,2 \%$ de la population au Brésil, dont la prévalence est augmentée en raison du vieillissement de la population (MUZY et al., 2021), le DM a causé un fardeau sur les caisses publiques, en plus de permettre également des coûts élevés pour effectuer le traitement de ses complications telles que : pied diabétique, maladies cardiovasculaires, amputation et autres. II convient de mentionner que le traitement pharmacologique a fait des progrès dans le contrôle du DM, cependant, l'éducation sanitaire en tant que forme de prévention doit être transmise à la population générale individuellement ou en groupe, en mettant l'accent sur le problème de la maladie et ses dommages à la santé ( SILVA, 2018).

En ce qui concerne les complications de la DS, le pied diabétique est également appelé par son état physiopathologique multiforme, caractérisé par des lésions qui proviennent des pieds de la personne atteinte de diabète de neuropathie dans $90 \%$ des cas, dans lesquels la maladie vasculaire périphérique et les déformations sont prédominantes (CUBAS et al., 2017).

De Sousa Mendonça; Moraes; Moura (2017) décrit que les lésions existantes dans le pied diabétique proviennent de la combinaison de deux ou plusieurs facteurs de risque qui peuvent être liés à des traumatismes intrinsèques tels que extrinsèques, associés à une neuropathie périphérique entraînant une perte de sensibilité, facilitant le risque de coupures; Maladie vasculaire périphérique résultant d'une réduction du flux sanguin dans les membres inférieurs et d'une altération biomécanique qui se produit lorsqu'il y a restriction des mouvements des articulations du pied et de la cheville.

On estime que l'incidence de l'ulcère du pied diabétique atteint $6,3 \%$ des patients diabétiques et sa prévalence approche les $10 \%$, et les personnes ayant un faible statut socio-économique sont les plus grandes victimes. L'amputation du pied diabétique est un autre problème et que dans le monde entier toutes les trois 
minutes, une personne se fait amputer un membre en raison de la DS (ARRUDA et al., 2021).

La bonne gestion des facteurs impliqués dans l'étiologie de la lésion existante dans le pied diabétique devient efficace lorsqu'elle est effectuée par l'infirmière, de sorte que l'infirmière doit être qualifiée et qualifiée pour effectuer la consultation infirmière afin de ne pas voir les changements dermatologiques, musculo-squelettiques, vasculaires et neurologiques dans le pied diabétique (FÉLIX et al., 2021).

Par conséquent, le soin des pieds diabétiques devient très important en santé publique et l'infirmière, en tant que professionnelle qui a sur ses prémisses les soins du patient, doit être attentive et qualifiée en ce qui concerne les nouvelles formes de traitement pour réduire les amputations. Face à ce scénario de taux alarmants, il est essentiel d'observer comment les infirmières se situent dans les cabinets de soins aux pieds diabétiques.

Compte tenu de ces preuves qui impliquent des chiffres alarmants en ce qui concerne le pied diabétique, le travail est basé sur la question problématique suivante: Quels soins les infirmières développent-elles chez les patients ayant des pieds diabétiques?

L'étude en question vise à prouver la prise en charge du pied diabétique liée à la pratique des infirmières.

\section{MÉTHODOLOGIE}

Pour cette étude, une revue intégrative de la littérature a été menée. Selon Mendes; Silveira et Galvão (2019), la méthode d'examen intégratif est développée selon des étapes bien définies; il s'agit de l'identification du thème et du choix de la question nordique; établir des critères d'inclusion et d'exclusion; l'identification d'études présélectionnées et sélectionnées; catégorisation des études sélectionnées; l'analyse et l'interprétation des résultats et la présentation du résumé de l'examen. 
La première étape de l'examen intégratif est la question nordique, qui mène au thème et aux objectifs énumérés. Par conséquent, la question qui se pose dans cet article est la suivante: Quels soins les infirmières développent-elles chez les patients ayant des pieds diabétiques?

Pour établir les critères d'inclusion ont été utilisés: articles publiés et disponibles à la lecture, car le sujet reflète l'infirmière dans leurs attributions, la base de données de BDENF, LILACS et SCIELO a été choisie, la délimitation temporelle était dans les années 2016 à 2020, c'est-à-dire au cours des cinq dernières années, et la langue portugaise a été choisie pour cette inclusion.

Les critères d'exclusion ont été supprimés: articles qui ont une duplicité, articles incomplets, actes, textes d'opinion, éditoriaux et lettres au lecteur, articles de revue, thèses, mémoires, textes complets, mais qui ne se rapportent pas au thème.

La période de collecte des données correspondait au mois de septembre 2021. Les descripteurs nationaux identifiés étaient : " soins infirmiers ", " pied diabétique ", " infirmière ", " diabète sucré ". L'opérateur booléen and a été utilisé pour associer les descripteurs dans la stratégie de recherche sur la plate-forme de base de données.

Pour la sélection des études sélectionnées et présélectionnées, une lecture approfondie des titres et des résumés des articles trouvés a été effectuée, puis une lecture plus complète tout au long de l'article, en recherchant dans ses résultats l'identification pour atteindre l'objectif proposé dans cet article.

Deux recherches ont été effectuées, la première a utilisé la stratégie de recherche suivante: soins infirmiers and pied diabétique and diabète sucré, avec cette stratégie, 186 articles ont été trouvés. Après avoir utilisé les filtres : texte intégral et disponible, base de données, langue portugaise et délimitation temporelle des cinq dernières années, il restait 24 articles à l'éligibilité. La deuxième recherche a été effectuée avec la stratégie suivante: infirmière and pied diabétique trouvant 95 articles. Après avoir utilisé les filtres, il restait 11 articles pour l'admissibilité. 
Les études ont été catégorisées à partir d'une image synoptique et l'analyse et la présentation des résultats ont été menées après la catégorisation qui a identifié et défini les noyaux de signification des études, et que de l'analyse thématique de Minayo (2012), il y a eu une discussion entre les auteurs trouvés.

Pour la synthèse des connaissances, une analyse des limites, de la représentativité de l'étude pour la communauté en général (pertinence) et des études futures identifiées au cours de cet article a été effectuée. Toutes les productions utilisées dans cette étude sont rigoureusement référencées selon les normes de l'Association brésilienne des normes techniques (ABNT).

\section{RÉSULTATS ET DISCUSSIONS}

Les articles restants pour l'admissibilité avec les deux stratégies totalisent 35 articles. Initialement, les articles de revue, les documents d'achèvement de cours, les lettres au lecteur, les rapports d'expérience, les études de cas, les articles en double ont été supprimés et que dans leurs titres, les résumés et la lecture dans leur intégralité n'ont pas apporté l'infirmière et ses soins comme thème au sujet, laissant ainsi 07 articles pour cette revue.

Pour une meilleure illustration des résultats trouvés, le tableau suivant comprendra la caractérisation des études sélectionnées. Les données se réfèrent au titre de l'article, à l'année de publication, à la base de données, à la revue, à l'emplacement, à l'objectif et aux résultats.

Table 1. Articles trouvés dans la base de données BDENF, LILACS et SCIELO sur les soins infirmiers pour les patients atteints de pieds diabétiques.

\begin{tabular}{|l|l|l|l|l|l|}
\hline $\begin{array}{l}\text { Titre/Année } \\
\text { de publication }\end{array}$ & $\begin{array}{l}\text { base } \\
\text { de } \\
\text { donnée } \\
\text { s }\end{array}$ & e & & Local & Résultats \\
\hline Conheciment & BDENF & Rev. & Comprendre & Stratégie de & Effectuer \\
\hline
\end{tabular}




\begin{tabular}{|c|c|c|c|c|c|}
\hline $\begin{array}{l}0 \text { do } \\
\text { enfermeiro } \\
\text { acerca dos } \\
\text { cuidados com } \\
0 \quad \text { pé } \\
\text { diabético } \\
2019\end{array}$ & & $\begin{array}{l}\text { enferm. } \\
\text { UFPE on } \\
\text { line }\end{array}$ & $\begin{array}{l}\text { les } \\
\text { connaissances } \\
\text { de l'infirmière } \\
\text { sur les soins } \\
\text { des pieds } \\
\text { diabétiques en } \\
\text { soins primaires }\end{array}$ & $\begin{array}{l}\text { santé } \\
\text { familiale/FS } \\
\text { E }\end{array}$ & $\begin{array}{l}\text { périodiqueme } \\
\text { nt un examen } \\
\text { physique des } \\
\text { pieds chez } \\
\text { les } \\
\text { diabétiques; } \\
\text { Utilisation } \\
\text { d'instruments } \\
\text { pour } \\
\text { l'évaluation } \\
\text { du pied } \\
\text { diabétique } \\
\text { Prendre soin } \\
\text { de soi aux } \\
\text { pieds }\end{array}$ \\
\hline $\begin{array}{l}\text { Riscos } \\
\text { associados à } \\
\text { mortalidade } \\
\text { em pacientes } \\
\text { atendidos em } \\
\text { um programa } \\
\text { de prevenção } \\
\text { do pé } \\
\text { diabético } \\
2018\end{array}$ & $\begin{array}{l}\text { LILACS } \\
/ \\
\text { BDENF }\end{array}$ & $\begin{array}{l}\text { Rev. } \\
\text { gaúch. } \\
\text { enferm }\end{array}$ & $\begin{array}{l}\text { Identifier chez } \\
\text { les patients } \\
\text { atteints de } \\
\text { diabète de type } \\
2 \quad \text { quels } \\
\text { changements } \\
\text { dans les pieds } \\
\text { seraient } \\
\text { associés aux } \\
\text { caractéristiques } \\
\text { démographique } \\
\text { s, cliniques, } \\
\text { biochimiques et } \\
\text { de traitement et } \\
\text { lesquels d'entre } \\
\text { eux }\end{array}$ & $\begin{array}{l}\text { Programme } \\
\text { éducatif } \\
\text { pour la } \\
\text { prévention } \\
\text { et le } \\
\text { traitement } \\
\text { du pied } \\
\text { diabétique } \\
\text { dans un } \\
\text { hôpital } \\
\text { général et } \\
\text { universitaire } \\
\text { de grande } \\
\text { complexité }\end{array}$ & $\begin{array}{l}\text { L'éducation } \\
\text { sanitaire sur } \\
\text { l'hygiène des } \\
\text { pieds, le } \\
\text { choix de } \\
\text { chaussures } \\
\text { appropriées, } \\
\text { la meilleure } \\
\text { conduite dans } \\
\text { les situations } \\
\text { d'urgence. } \\
\text { Soins } \\
\text { systémiques } \\
\text { tels que ne } \\
\text { pas fumer, } \\
\text { maintenir un }\end{array}$ \\
\hline
\end{tabular}




\begin{tabular}{|c|c|c|c|c|c|}
\hline & & & $\begin{array}{l}\text { augmenteraient } \\
\text { le risque de } \\
\text { mortalité }\end{array}$ & & $\begin{array}{l}\text { bon contrôle } \\
\text { glycémique, } \\
\text { contrôler les } \\
\text { taux de } \\
\text { lipides } \\
\text { sanguins et } \\
\text { l'utilisation } \\
\text { correcte des } \\
\text { médicaments }\end{array}$ \\
\hline $\begin{array}{l}\text { Condutas dos } \\
\text { enfermeiros } \\
\text { da atenção } \\
\text { primária no } \\
\text { cuidado a } \\
\text { pessoas com } \\
\text { pé diabético } \\
2017\end{array}$ & BDENF & $\begin{array}{l}\text { Rev. } \\
\text { enferm. } \\
\text { UFPE on } \\
\text { line }\end{array}$ & $\begin{array}{l}\text { Connaître les } \\
\text { actions des } \\
\text { infirmières de } \\
\text { soins primaires } \\
\text { dans les soins } \\
\text { aux personnes } \\
\text { atteintes de } \\
\text { diabète sucré } \\
\text { (DS) lié au pied } \\
\text { diabétique }\end{array}$ & $\begin{array}{l}\text { Stratégie de } \\
\text { santé } \\
\text { familiale }\end{array}$ & $\begin{array}{l}\text { Fréquence et } \\
\text { planification } \\
\text { des } \\
\text { consultations, } \\
\text { dynamique du } \\
\text { service, } \\
\text { critères } \\
\text { d'examen des } \\
\text { pieds, soins } \\
\text { personnels }\end{array}$ \\
\hline $\begin{array}{l}\text { Ações do } \\
\text { enfermeiro na } \\
\text { prevenção do } \\
\text { pé diabético: } \\
\text { o olhar da } \\
\text { pessoa com } \\
\text { diabetes } \\
\text { mellitus } \\
2017\end{array}$ & $\begin{array}{l}\text { LILACS } \\
/ \\
\text { BDENF }\end{array}$ & $\begin{array}{l}\text { Rev. } \\
\text { Pesqui. } \\
\text { (Univ. } \\
\text { Fed. } \\
\text { Estado } \\
\text { Rio J., } \\
\text { Online) }\end{array}$ & $\begin{array}{l}\text { Enquête sur les } \\
\text { actions menées } \\
\text { par les } \\
\text { infirmières dans } \\
\text { la prévention } \\
\text { du pied } \\
\text { diabétique du } \\
\text { point de vue de } \\
\text { la personne } \\
\text { atteinte de DS. }\end{array}$ & $\begin{array}{l}\text { Projet } \\
\text { d'extension } \\
\text { de } \\
\text { l'université }\end{array}$ & $\begin{array}{l}\text { Des actions } \\
\text { spécifiques, } \\
\text { qui impliquent } \\
\text { correctement } \\
\text { l'examen des } \\
\text { pieds, le } \\
\text { dialogue } \\
\text { communicatif, } \\
\text { l'éducation à } \\
\text { la santé. }\end{array}$ \\
\hline $\begin{array}{l}\text { Alterações } \\
\text { nos pés do }\end{array}$ & $\begin{array}{l}\text { LILACS } \\
/\end{array}$ & $\begin{array}{l}\text { Esc. } \\
\text { Anna }\end{array}$ & $\begin{array}{l}\text { Caractériser le } \\
\text { profil }\end{array}$ & Hôpital & $\begin{array}{l}\text { Renforcer les } \\
\text { liens avec ces }\end{array}$ \\
\hline
\end{tabular}




\begin{tabular}{|c|c|c|c|c|c|}
\hline $\begin{array}{l}\text { idoso } \\
\text { hospitalizado: } \\
\text { um olhar } \\
\text { cuidadoso da } \\
\text { enfermagem } \\
2017\end{array}$ & BDENF & $\begin{array}{l}\text { Nery } \\
\text { Rev. } \\
\text { Enferm }\end{array}$ & $\begin{array}{l}\text { pédologique } \\
\text { des personnes } \\
\text { âgées } \\
\text { hospitalisées } \\
\text { dans les } \\
\text { services d'un } \\
\text { hôpital } \\
\text { universitaire; } \\
\text { identifier les } \\
\text { demandes de } \\
\text { soins des pieds } \\
\text { des patients } \\
\text { âgés } \\
\text { hospitalisés; et } \\
\text { d'analyser les } \\
\text { possibilités } \\
\text { d'actions des } \\
\text { infirmières } \\
\text { auprès de ces } \\
\text { personnes } \\
\text { âgées. }\end{array}$ & & $\begin{array}{l}\text { patients } \\
\text { diabétiques } \\
\text { afin qu'ils } \\
\text { viennent au } \\
\text { poste pour } \\
\text { effectuer } \\
\text { fréquemment } \\
\text { des examens } \\
\text { du pied } \\
\text { Formation et } \\
\text { qualification } \\
\text { pour aider } \\
\text { ces problèmes, } \\
\text { priter de plus } \\
\text { grandes } \\
\text { complications } \\
\text { et réduire les } \\
\text { demandes de } \\
\text { soins futures }\end{array}$ \\
\hline $\begin{array}{l}\text { Atuação dos } \\
\text { enfermeiros } \\
\text { da estratégia } \\
\text { saúde da } \\
\text { família na } \\
\text { prevenção do } \\
\text { pé diabético } \\
2016\end{array}$ & $\begin{array}{l}\text { LILACS } \\
/ \\
\text { BDENF }\end{array}$ & $\begin{array}{l}\text { Rev. } \\
\text { Pesqui. } \\
\text { (Univ. } \\
\text { Fed. } \\
\text { Estado } \\
\text { Rio J., } \\
\text { Online) ; }\end{array}$ & $\begin{array}{l}\text { Identifier les } \\
\text { conseils fournis } \\
\text { par les } \\
\text { infirmières aux } \\
\text { personnes } \\
\text { atteintes de DS } \\
\text { sur les soins } \\
\text { des pieds; } \\
\text { Étudier la } \\
\text { fréquence de }\end{array}$ & $\begin{array}{l}\text { Stratégie de } \\
\text { santé } \\
\text { familiale }\end{array}$ & $\begin{array}{l}\text { Éducation } \\
\text { sanitaire pour } \\
\text { les } \\
\text { diabétiques, } \\
\text { examen des } \\
\text { pieds des } \\
\text { personnes } \\
\text { atteintes de } \\
\text { DS, formation } \\
\text { de groupes }\end{array}$ \\
\hline
\end{tabular}




\begin{tabular}{|c|c|c|c|c|c|}
\hline & & & $\begin{array}{l}\text { l'examen du } \\
\text { pied et les } \\
\text { aspects } \\
\text { évalués; vérifier } \\
\text { quelles activités } \\
\text { d'éducation à la } \\
\text { santé } \\
\text { infirmières les } \\
\text { effectuent pour } \\
\text { les personnes } \\
\text { atteintes de } \\
\text { DS. }\end{array}$ & & $\begin{array}{l}\text { de } \\
\text { coexistence, } \\
\text { pansements } \\
\text { dans des } \\
\text { techniques } \\
\text { appropriées } \\
\text { au type de } \\
\text { blessure au } \\
\text { pied. }\end{array}$ \\
\hline $\begin{array}{l}\text { A perspectiva } \\
\text { da } \\
\text { vulnerabilidad } \\
\text { e na } \\
\text { avaliação do } \\
\text { pé diabético } \\
\text { sob a ótica } \\
\text { de } \\
\text { enfermeiros } \\
2016\end{array}$ & BDENF & $\begin{array}{l}\text { Cogitare } \\
\text { enferm }\end{array}$ & $\begin{array}{l}\text { Écrivez les } \\
\text { conditions } \\
\text { sociales } \\
\text { déterminantes } \\
\text { de la santé, } \\
\text { identifiées par } \\
\text { les infirmières } \\
\text { comme } \\
\text { pertinentes } \\
\text { pour } \\
\text { l'établissement } \\
\text { des } \\
\text { vulnérabilités } \\
\text { de la personne } \\
\text { atteinte de } \\
\text { diabète sucré. }\end{array}$ & $\begin{array}{l}\text { Infirmières } \\
\text { choisies au } \\
\text { hasard à } \\
\text { Curitiba }\end{array}$ & $\begin{array}{l}\text { Stimulez le } \\
\text { diabétique à } \\
\text { aller pour une } \\
\text { évaluation } \\
\text { nutritionnelle } \\
\text { et à suivre le } \\
\text { régime } \\
\text { alimentaire. }\end{array}$ \\
\hline
\end{tabular}

Source : Auteur. 
L'étude dans ses objectifs mentionne trois types de soins effectués par les infirmières pour les patients atteints de pieds diabétiques: le premier qui a le plus émergé est basé sur des actions visant l'éducation à la santé en tant que principe fondamental de la prévention des maladies et de ses complications, le deuxième soin est basé sur des techniques d'évaluation du pied chez les diabétiques comme moyen de survenue de complications et le troisième soins se concentre sur les infirmières en tant que stimulateur et motivateur patients diabétiques à leurs soins personnels avec leurs pieds. Ces trois précautions seront abordées dans leurs thématiques selon les études sélectionnées.

\subsection{L'ÉDUCATION À LA SANTÉ COMME FORME DE PRÉVENTION}

Par conséquent, à partir de l'éducation à la santé, le patient peut comprendre les raisons pour lesquelles le processus de cicatrisation des plaies est lent, les raisons des principales complications et surtout les connaissances liées à la prévention des risques. Les infirmières devraient être en mesure de reconnaître les besoins du patient et ainsi contribuer à des diagnostics infirmiers précis et sécuritaires liés à la plaie à son stade.

Scain; Franzen; Hirakata (2018) rapporte que les infirmières ont maintenu leurs efforts pour effectuer une éducation à la santé avec les patients, deviennent persistantes et insistantes pour poursuivre le processus éducatif chez les patients ayant des pieds diabétiques, utiliser la créativité avec le développement de technologies éducatives telles que des dossiers, des vidéos, ainsi que d'être disponibles pour poser des questions sur une condition anormale avec le patient à partir de technologies de médias sociaux telles que Whatsapp / téléphone.

Camillo et al. (2016) mentionne que le concept d'éducation à la santé va au-delà de l'apport de données ou même de la levée des doutes qui surgissent, les auteurs considèrent l'éducation à la santé comme un processus systématique, continu et permanent, visant à la formation et au développement de la conscience critique du citoyen, à travers la recherche de solutions collectives aux problèmes rencontrés et leur « participation réelle » à l'exercice du contrôle social. 
Vargas et al. (2017) fait de l'éducation à la santé l'une des principales finalités des attributions de soins de l'infirmière, les auteurs renforcent que l'éducation à la santé favorise une attitude plus proactive pour le patient, démontrant que le patient doit être en confiance dans la prise en charge du pied diabétique.

L'autonomie et la sécurité des soins ont également été soulignées comme résultats de l'éducation à la santé effectuée par les infirmières dans l'étude de Pereira et al. (2017), les auteurs rapportent que l'éducation à la santé devient le principal mécanisme de prévention contre les maladies et leurs complications, en relation avec le pied diabétique, l'éducation à la santé favorise la sécurité des patients, la confiance et l'autonomie.

La démonstration de l'efficacité de l'éducation à la santé a été identifiée dans une étude descriptive de l'approche quantitative dans laquelle elle a montré que l'éducation à la santé apportait aux patients une connaissance théorique de la maladie en respectant la culture et la croyance des patients, et que la promotion des processus éducatifs se déroule non seulement ensemble, mais de manière individualisée, éliminer les doutes qui imprègnent le patient lors de la consultation infirmière ou même dans un dialogue informel entre le patient et le professionnel (DE OLIVEIRA et al., 2016).

Mais l'éducation à la santé n'est pas seulement identifiée comme un processus de soins aux patients, les infirmières doivent également être attentives à la formation et aux qualifications, qui peuvent également être considérées comme une éducation à la santé d'une manière plus technique. Silva; Santo et Chibante (2017) affirment que les infirmières devraient toujours être mises à jour vers de nouvelles façons de prendre soin des pieds diabétiques, car les processus de traitement sont de plus en plus étudiés dans la littérature scientifique et apportent des innovations pour la guérison ou même la stadification des plaies.

Par conséquent, l'éducation à la santé, la formation continue et la formation continue sont des formes de soins que les infirmières utilisent pour améliorer le tableau clinique des patients atteints de pieds diabétiques. 


\subsection{TECHNIQUES D’ÉVALUATION DU PIED DIABÉTIQUE}

Les connaissances technico-scientifiques acquises au cours des phases de formation infirmière et dans le contexte de la formation continue et permanente sont des facteurs pertinents pour le traitement du pied diabétique, il appartient donc à l'infirmière de rechercher des connaissances pour effectuer des techniques d'évaluation sûres pour les patients atteints de diabète en tant que méthode de prévention et pour les patients atteints de pied diabétique en tant que mesures de prévention des complications.

Arruda et al. (2019) indique que l'examen physique des pieds diabétiques sont des procédures effectuées par des infirmières afin d'éviter d'éventuelles complications telles que: le développement d'ulcères et d'amputations des membres inférieurs. Par conséquent, l'ignorance de cette technique induit des taux plus élevés de complications. Les auteurs ont mentionné souligner à un autre moment qu'en plus de la technique qui n'est pas utilisée en raison du manque de connaissance de l'infirmière, les conditions de travail influencent également la non-performance de la technique d'évaluation, ces conditions sont qualifiées par les auteurs d'infrastructure inadéquate et de demande de soins excessifs.

Vargas et al. (2017) apporte dans son étude un contexte similaire à l'étude mentionnée précédemment et ajoute que les infirmières ont besoin de plus de formation sur les techniques d'évaluation liées au pied diabétique, les infirmières signalent également que la direction n'offre pas de matériel pour effectuer ces techniques et parlent de demandes excessives.

Pereira et al. (2017) estime que des mesures spécifiques devraient être prises pour remodeler le système de soins pour les patients atteints du pied diabétique, ces actions devraient être liées au-delà de l'examen du pied, présentant une communication efficace et une éducation à la santé.

Leal et al. (2016) considère que les infirmières peuvent avoir une ignorance du sujet en raison de plusieurs facteurs et l'un d'eux est souligné par les auteurs comme la 
forte demande de soins. Cette exigence implique un épuisement physique et mental du professionnel, ce qui manque de temps pour la qualification professionnelle. Dans un autre sens, les auteurs mentionnent que la demande excessive pour un problème donné permet au professionnel de faire place à une éducation permanente à la santé, dans laquelle ce type de formation propose des transformations dans les pratiques professionnelles en fonction des problèmes existants dans le processus de leur apprentissage du travail pour refléter la pratique dans leur réalité.

Les soins infirmiers ont pour fonction de traiter les maladies de la peau et ses maladies adjacentes. En effet, les infirmières ont dans leurs attributions les soins au patient et la nécessité d'étendre ces soins associés aux maladies liées à la peau (DA SILVA BRANDAO et al., 2016; SA et al., 2016).

\subsection{L'INFIRMIÈRE MÉDIATRICE POUR LES SOINS PERSONNELS DES PATIENTS ATTEINTS DE PIEDS DIABÉTIQUES}

Parler de soins personnels fait référence à toute action dérivée de l'acte quotidien de l'être humain. Quant aux soins personnels Arruda et al. (2019) rapportent que les patients atteints du pied diabétique ont tendance à générer un grand impact économique, social, culturel, émotionnel et biologique, ce qui favorise une plus grande dépendance aux autres pour aider dans les activités de la vie quotidienne et un impact sur leurs soins personnels et leur qualité de vie.

Scain; Franzen et Hirakata (2018) rapportent que le patient doit être conscient de la performance de ses propres activités et que les soins personnels doivent être continus, l'une des façons d'effectuer des soins personnels cités par les auteurs était: Hygiène des pieds; le choix de chaussures appropriées; évitez de marcher pieds nus et autres.

À Vargas et al. (2017) apporte comme auto-soin le maintien du contrôle glycémique, la participation proactive des patients à la réalisation d'examens des pieds, la thérapie, la restriction absolue à l'utilisation de tabac et de boissons, la nutrition, 
l'exercice physique, l'activité physique et d'autres formes qui envisagent l'étude précédemment référencée.

Un moyen d'atteindre que le patient peut viser à prendre soin de lui-même est cité par les auteurs Silva; Santo et Chibante (2017) comme le renforcement des liens entre le patient et le professionnel de la santé. Ce renforcement favorise la sécurité et la crédibilité du patient que l'auto-soin des pieds de manière continue et systématique, plus les risques de complications sont faibles.

L'autonomie et la sécurité démontrées par les patients atteints de pied diabétique minimisent les dommages et les risques de complications. Un patient qui est impliqué dans le traitement de sa maladie a tendance à élargir ses connaissances à la nondépendance d'autres personnes, en articulant dans ses activités les soins personnels comme une forme de motivation et de démonstration de capacité face aux adversités qui impliquent le processus santé-maladie (DE OLIVEIRA et al., 2016).

À Fatima Bento et al. (2016), les patients aux pieds diabétiques souffrent déjà de stigmatisation de leur état, ce qui rend difficile pour eux d'avoir des soins adéquats, car les conditions qui impliquent de se distancier du bureau de santé, des difficultés à se mobiliser, peu de connaissances sur la maladie, permettent des soins inadéquats et l'évolution des complications.

Cependant, Nascimento et al. (2016) mentionnent que lorsque des soins qualifiés et humanisés sont fournis par des comportements efficaces, avec un accès facile à des services de santé auxquels toutes les actions peuvent être intégrées à tous les niveaux de soins, les autosoins sans risque de complications.

\section{CONCLUSION}

Cette étude a porté sur les soins des infirmières et des professionnels dans la prestation des soins et des soins aux patients atteints du pied diabétique. Les études 
de soins pertinentes ont identifié ce qui a conduit à la réalisation de trois thèmes pour la discussion des résultats trouvés.

L'objectif de l'étude visait à mettre en évidence les soins fournis aux infirmières aux patients atteints de pieds diabétiques, il est souligné que les soins des infirmières et non d'autres catégories de soins infirmiers ont été soulignés. Les études ont démontré que l'éducation à la santé était le premier soin, ce qui ne peut être contredit, car la littérature souligne que l'éducation à la santé est primordiale pour la prévention des maladies.

Un autre type de soins se reflète dans les connaissances du professionnel sur le pied diabétique, sur les besoins extrêmes et les grandes préoccupations du système de santé, car les articles ont apporté des réflexions selon lesquelles les infirmières devraient être plus qualifiées pour diriger les soins avec plus d'excellence vers le patient.

Un soin important également souligné par les études et qui est dirigé directement avec la formation permanente ou continue des professionnels sont les techniques d'examen physique, dans ce type de soins, l'infirmière devrait être en mesure d'effectuer l'examen des pieds diabétiques judicieusement et en toute sécurité, en plus d'encourager les patients à se rendre à l'examen plus fréquemment.

En ce qui concerne les soins liés aux soins personnels du patient, il a été reflété que l'hygiène des pieds, le contrôle glycémique, le suivi continu avec examen des pieds, l'utilisation de chaussures appropriées et d'autres intensifient une plus grande prévention contre les complications parmi lesquelles l'amputation des membres.

Il est donc conclu que l'étude a identifié plusieurs formes de soins dispensés par les infirmières et que toutes ces formes sont importantes pour la continuité du traitement et la prévention et les complications, cependant, il appartient à l'infirmière d'être un professionnel qui valorise la qualité de ses soins en fonction de concepts théoriques qui conduisent à une formation fréquente. 


\section{RÉFÉRENCES}

AMERICAN DIABETES ASSOCIATION. Standards of medical care in diabetes. Diabetes Care, v. 40, n. suppl 1, p. s1-s128, 2017.

ARRUDA, Cecilia et al. Tecnologia educativa para cuidados e prevenção do pé diabético/Educational technology for care and prevention of diabetic foot ulcers. Ciência, Cuidado e Saúde, v. 20, 2021.

ARRUDA, Luana Savana Nascimento de Sousa et al. Conhecimento do enfermeiro acerca dos cuidados com o pé diabético. Rev. enferm. UFPE on line, p. [1-8], 2019.

ASCHNER M, Pablo et al. Clinical practice guideline for the prevention, early detection, diagnosis, management and follow up of type 2 diabetes mellitus in adults. Colomb. Med., Cali, v. 47, n. 2, p. 109-130, junho. 2016.

CAMILLO, Bibiana Schultz et al. Ações de educação em saúde na atenção primária a gestantes e puérperas: revisão integrativa. Revista de enfermagem UFPE on line-ISSN: 1981-8963, v. 10, n. 6, p. 4894-4901, 2016.

CUBAS, Marcia Regina et al. Pé diabético: orientações e conhecimento sobre cuidados preventivos. Fisioterapia em movimento, v. 26, n. 3, 2017.

DE FÁTIMA BENTO, Leandra et al. A perspectiva da vulnerabilidade na avaliação do pé diabético sob a ótica de enfermeiros. Cogitare Enfermagem, v. 21, n. 1, 2016.

DE OLIVEIRA, Patrícia Simplício et al. Atuação dos enfermeiros da Estratégia Saúde da Família na prevenção do pé diabético Practice nurse family health strategy in the prevention of diabetic foot. Revista de Pesquisa Cuidado é Fundamental Online, v. 8, n. 3, p. 4841-4849, 2016.

DE SOUZA MENDONÇA, Sarah; MORAIS, Juliana de Sant'Anna; DE MOURA, Maria Catarina Gomes Gadelha. Proposta de um protocolo de avaliação 
fisioterapêutica para os pés de diabéticos. Fisioterapia em Movimento, v. 24, n. 2, 2017.

FELIX, Lidiany Galdino et al. Conhecimento de enfermeiros da atenção primária antes e após intervenção educativa sobre pé diabético. Revista Gaúcha de Enfermagem, v. 42, 2021.

LEAL, Maria do Carmo et al. Atenção ao pré-natal e parto em mulheres usuárias do sistema público de saúde residentes na Amazônia Legal e no Nordeste, Brasil 2010. Rev. Bras. Saude Mater. Infant. Recife, v. 15, n. 1, p. 91-104, Mar. Available from \&lt; http://www.scielo.br/scielo.php?script=sci_arttext\&amp;pid=S151938292015000100091\&amp; Ing=en\&amp;nrm=iso\&gt;.

MENDES, Karina Dal Sasso; SILVEIRA, Renata Cristina de Campos Pereira; GALVÃO, Cristina Maria. Uso de gerenciador de referências bibliográficas na seleção dos estudos primários em revisão integrativa. Texto \& ContextoEnfermagem, v. 28, 2019.

MINAYO, Maria Cecília de Souza. Análise qualitativa: teoria, passos e fidedignidade. Ciência \& saúde coletiva, v. 17, p. 621-626, 2012.

MUZY, Jéssica et al. Prevalência de diabetes mellitus e suas complicações e caracterização das lacunas na atenção à saúde a partir da triangulação de pesquisas. Cadernos de Saúde Pública, v. 37, 2021.

NASCIMENTO, Vagner Ferreira do et al. Percepção de puérperas sobre as primeiras consultas de pré-natal no interior de Mato Grosso. Rev. enferm. UFPI, v.5, n. 1, p. 46-51, 2016.

ORGANIZAÇÃO MUNDIAL DE SAÚDE. Definition, diagnosis and classification of diabetes Mellitus and its complications. Geneva: OMS, 1999. Disponível em: https://apps.who.int/iris/bitstream/handle/10665/66040/WHO_NCD_NCS_99.2.pdf?s equence $=1$ \&isAllowed $=\mathrm{y}$ 
PEREIRA, Laiane de Fátima et al. Ações do enfermeiro na prevenção do pé diabético: o olhar da pessoa com diabetes mellitus. Rev. pesqui. cuid. fundam. (Online), p. 1008-1014, 2017.

SCAIN, Suzana Fiore; FRANZEN, Elenara; HIRAKATA, Vânia Naomi. Riscos associados à mortalidade em pacientes atendidos em um programa de prevenção do pé diabético. Revista Gaúcha de Enfermagem, v. 39, 2018.

SILVA, Caline Oliveira da. Análise da qualidade de vida em indivíduos com Diabetes Mellitus Tipo 1. 2017. 34 f. Trabalho de conclusão de curso (Bacharelado em Farmácia) -Universidade de Brasília, Brasília, 2017. Disponível em: https://bdm.unb.br/handle/10483/19301

SILVA, Joziane Santos da; SANTO, Fátima Helena do Espírito; CHIBANTE, Carla Lube de Pinho. Alterações nos pés do idoso hospitalizado: um olhar cuidadoso da enfermagem. Escola Anna Nery, v. 21, 2017.

SOCIEDADE BRASILEIRA DE DIABETES. Tratamento e acompanhamento do diabetes Mellitus: Diretrizes da Sociedade Brasileira de Diabetes. 3.ed. Itapevi, SP: A. Araújo Silva Farmacêutica; 2017/2018. Disponível em: http://www.diabetes.org.br/ profissionais/images/2017/diretrizes/diretrizes-sbd-20172018.pdf.

VARGAS, Caroline Porcelis et al. Condutas dos enfermeiros da atenção primária no cuidado a pessoas com pé diabético. Rev. enferm. UFPE on line, p. 4535-4545, 2017.

Soumis: Octobre, 2021.

Approbation : Décembre 2021. 